\title{
Appropriate New Structure Selection for a Specific Function of Architecture
}

\author{
MARYAM GHORBANZADEH \\ Department of Architecture, Faculty of Art, University of Bojnord, Bojnord, Iran. \\ http://dx.doi.org/10.12944/CWE.11.Special-Issue1.05
}

(Received: July, 2016; Accepted: August, 2016)

\begin{abstract}
Today, with the introduction of new structural systems to the construction industry, making the decision to use this new system is not easy. Due to the unique characteristics that each of the systems have and also with regard to the specific circumstances of each project, it might be possible that each of these systems find top priority to common structural systems of the country. In this article by using value engineering and scoring criteria tailored to each user and scoring structures (due to the specific characteristics of each structure) the way to choose the optimal structural system for every user and for every function is discussed. Finally, for hypothetical use, the College of Architecture, after processing, Space frame structure and the folded plate as optimized structure, was selected among other structures.
\end{abstract}

Keywords: Optimal structure, criterion, construction systems, scoring.

\section{INTRODUCTION}

To achieve the necessary harmony between architectural design and its construction requires analysis of a variety of structures and choosing the most appropriate one for each function.

Due to extensive requirements and the need to improve the quality of building as is common in most large countries the best industrial production methods and the most use of new structures should be considered. So that our architecture in Iran needs to change from traditional methods to modern methods of building design approaches.

Familiarity with a variety of structural systems for students and engineers of architecture and civil engineering is critical today. That unfortunately it has not been considered in educational programs for the civil and architecture majors of the Iran's universities.
The variety of construction subjects and access to new technology has helped the architects to design new buildings, very creatively. (Heydary, 2012)

But the quality and condition of using the technology in the creation of architecture has always been considered as controversial question. (Asefy, 2013)

Knowing the fact that use of new structures is a new topic in Iran, there is always the question in the minds of the vast majority of structure designer that which specific projects will satisfy from all aspects (aesthetic, functional, physical, etc.) needs of the user.

Scoring process of criteria and structure is an appropriate solution for selecting optimal system for each user to avoid the interference of designer's taste in the projects. 
For this purpose, the method was tried to quantify the quality to extract effective factors in architectural projects and with scoring process optimal structure is selected. ${ }^{1}$

It is noteworthy that this process stays quite systematic in a new study effort so that in the article "A KNOWLEDGE-BASED EXPERT SYSTEM FOR SELECTION OF APPROPRIATE STRUCTURAL SYSTEMS FOR LARGE SPANS," written by Mahmoud Golabchi deals with this subject:

"The progress of each smart system needs choosing the right tools. This subject depends on factors like: the type of work that is expected to be done by smart systems, the needs of users, the benefit and enjoyment of hardware and software resources available for development. The method of "smart systems of structural choice ${ }^{2}$ will follow three main steps:

- Comparison Science

- Application System

- Evaluation System" (Golabchi, 2008)

How to choose the optimal structures for architectural applications

Structure is a factor that can lead to disagreement between the architect and the structural engineer. Today, a good architect should have a general knowledge about the behavior of the structures. In addition, he is an artist and should be bound by the principles and standards of architectural design aesthetics. (Golabchi, 1999)

Today, with the introduction of new structural systems to the industry, and with all the benefits of new structures which was mentioned in the earlier sections, decision to use this new system is logical and self-evident. Due to the unique characteristics that each of these systems have and also according to the specific circumstances of each project, these systems may each find priority to common systems instrument. (Hesami, 2013)

The selection process along with architectural structures ${ }^{1}$

The selections process has the following steps:

1. Selection a structural system

2. Preliminary design
3. Detailed or analytical design (design analysis)

In this paper, specifically the selection of the structural system, the first step, is analyzed. This is done according to the following process:

\section{Function definition}

What is the favorable function?

At this point it is necessary to know the type

of use.

The definition of Initial criteria, constraints, functional requirements

Criteria of each design is defined in three groups:

The criteria of each plan is defined in three groups:

The Proposed criteria common to all applications of the project includes the following:

Cost

The initial cost of the project (cost of structures, materials and labor), current costs (maintenance, heating, cooling, ventilation, lighting, etc.), costs, after work (dismantling, demolition, etc.).

\section{Aesthetic}

architecture

$$
\text { Exterior architecture and interior }
$$

Minimum obstruction

Simplicity

Long Design life

Flexibility in use

Time of constructing

Specific criteria for the project, is the following:

The possibility of developing

The possibility of temporary use

Retractable roof

Acoustic 1-2-2-5 absorbing dynamic forces 1-2-2-6 minimum temperature effect

Functional requirements and constraints, including the following:

Span

Area should be covered 1-2-3-3 shape, geometry (square, rectangle, polygon, circle, ellipse, etc.)

Designing Time(duration of design)

Design life , 1-2-3-6 climate condition 1-2-3-7 Period

of construction and ...

Knowledge of types of building systems 
Scoring structures without considering the usage and on the basis of inherent structural features ${ }^{1}$

A mistake that occurs in most projects is the designer tastes that interfere in this important process. To avoid this mistake, the first step is to define and determine the priorities and criteria of the project design. Otherwise, the implementation of project might be prolonged, costs might increase, lack of required qualities for projects and other such

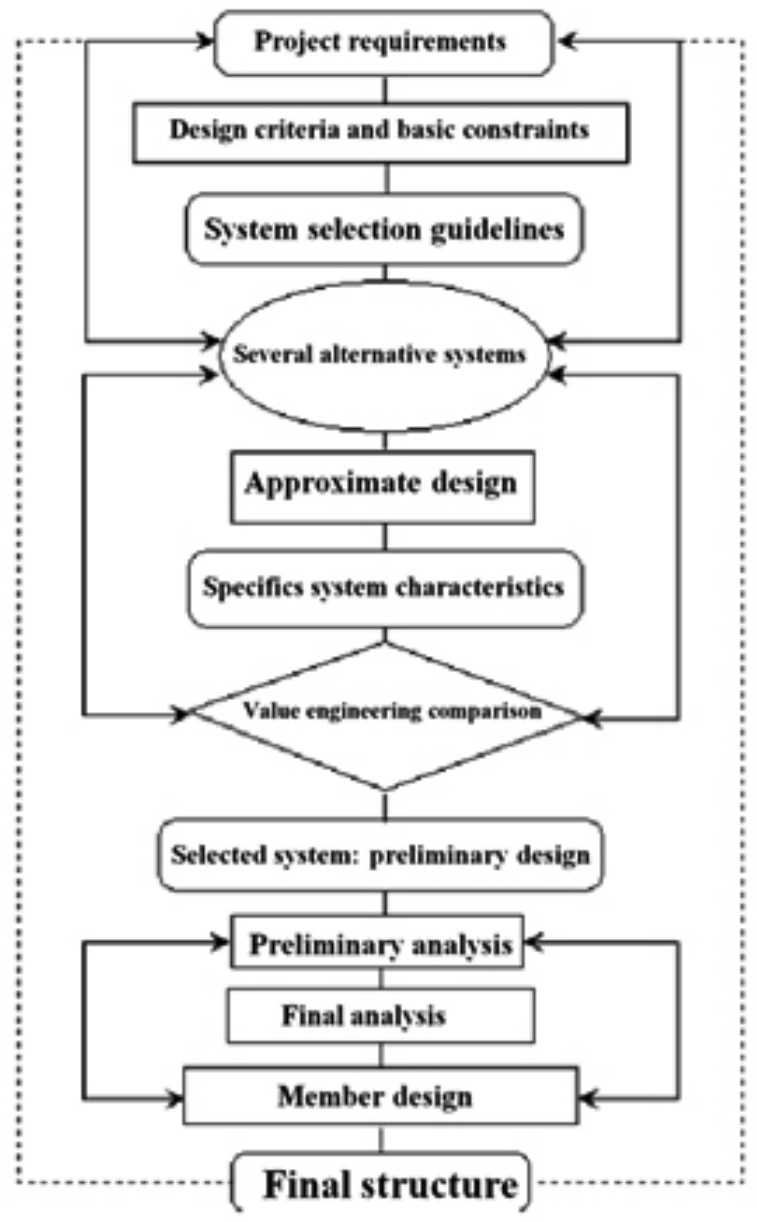

Fig. 1: Systematic design model, (Golabchi, 2008)

Table 1: Types of building systems

\begin{tabular}{|c|c|c|c|c|}
\hline $\begin{array}{l}\text { Truss } \\
\text { structures }\end{array}$ & $\begin{array}{l}\text { Grid } \\
\text { structures }\end{array}$ & $\begin{array}{l}\text { Pressure } \\
\text { structures }\end{array}$ & $\begin{array}{l}\text { Tension } \\
\text { structures }\end{array}$ & $\begin{array}{l}\text { Shell } \\
\text { structures }\end{array}$ \\
\hline $\begin{array}{l}\text { Truss } \\
\text { Space frames } \\
\text { Geodesic domes }\end{array}$ & $\begin{array}{l}\text { Columns } \\
\text { Beams and slabs } \\
\text { Frames }\end{array}$ & $\begin{array}{l}\text { Arches } \\
\text { Vaults }\end{array}$ & $\begin{array}{l}\text { Cable structures } \\
\text { Membrane structures } \\
\text { Pneumatic structures } \\
\text { (Air supporte\& air inflated } \\
\text { structures) } \\
\text { Tensegrity }\end{array}$ & $\begin{array}{l}\text { Thin shells } \\
\text { Folded plates }\end{array}$ \\
\hline
\end{tabular}


Table 2: Scoring structures with consideration to inherent property (7 tables)

\begin{tabular}{|c|c|}
\hline Maintenance & Types of structures \\
\hline Possible problem & Space frame \\
\hline Air supported: Normally expensive, possible problem & \multirow[t]{2}{*}{ Pneumatic } \\
\hline Air Inflated: Possible problem & \\
\hline Normally expensive & Cable \\
\hline Normally problem & Folded plate \\
\hline Possible problem: depend of materials of tent \& expecting wind forces & Membrane \\
\hline $\begin{array}{c}\text { Possible problem } \\
3\end{array}$ & Tensegrity \\
\hline $\begin{array}{l}\text { Normally expensive: with envision of bending \& propulsion } \\
\qquad 2\end{array}$ & Arch \\
\hline $\begin{array}{c}\text { Normally expensive: with envision of bending \& propulsion } \\
2\end{array}$ & Vault \\
\hline Possible problem: requining to especial envision to moisture \& fire & Truss \\
\hline Possible problem: requiring to especial envision to bracing & Frame \\
\hline Normally problem & $\begin{array}{l}\text { Hyperbolic parabolic } \\
\text { shell }\end{array}$ \\
\hline Normally problem & Cylindrical shell \\
\hline$\underset{3}{\text { Normally possible }}$ & Geodesic dome \\
\hline Normally problem & Grid \\
\hline
\end{tabular}

\begin{tabular}{|c|c|c|c|}
\hline Heaviness & $\begin{array}{c}\text { Time of } \\
\text { construction }\end{array}$ & $\begin{array}{c}\text { Easy of } \\
\text { construction }\end{array}$ & Types of structures \\
\hline $\begin{array}{l}\text { Light } \\
3\end{array}$ & Very short & $\begin{array}{c}\text { Very easy } \\
4\end{array}$ & Space frame \\
\hline$\underset{4}{\text { Very light }}$ & $\begin{array}{c}\text { Very short } \\
4\end{array}$ & $\begin{array}{c}\text { Rather difficult } \\
2\end{array}$ & Pneumatic \\
\hline$\underset{4}{\text { Very light }}$ & $\begin{array}{c}\text { Short } \\
3\end{array}$ & $\begin{array}{l}\text { Rather difficult } \\
2\end{array}$ & Cable \\
\hline $\begin{array}{c}\text { Heavy } \\
2\end{array}$ & $\underset{2}{\text { Long }}$ & $\begin{array}{c}\text { Easy } \\
3\end{array}$ & Folded plate \\
\hline$\underset{4}{\text { Very light }}$ & $\begin{array}{c}\text { Short } \\
3\end{array}$ & Rather difficult & Membrane \\
\hline $\begin{array}{l}\text { Light } \\
3\end{array}$ & $\begin{array}{c}\text { Long } \\
1\end{array}$ & $\begin{array}{c}\text { Difficult } \\
1\end{array}$ & Tensegrity \\
\hline $\begin{array}{c}\text { Very heavy } \\
1\end{array}$ & $\begin{array}{c}\text { Rather long } \\
2\end{array}$ & Rather difficult & Arch \\
\hline $\begin{array}{c}\text { Very heavy } \\
1\end{array}$ & Rather long & Rather difficult & Vault \\
\hline $\begin{array}{l}\text { Light } \\
3\end{array}$ & $\begin{array}{c}\text { Very short } \\
4\end{array}$ & Very easy & Truss \\
\hline $\begin{array}{c}\text { Heavy } \\
2\end{array}$ & $\begin{array}{c}\text { Short } \\
3\end{array}$ & $\begin{array}{l}\text { Rather difficult } \\
2\end{array}$ & Frame \\
\hline $\begin{array}{c}\text { Heavy } \\
2\end{array}$ & $\begin{array}{c}\text { Long } \\
2\end{array}$ & $\begin{array}{c}\text { Difficult } \\
1\end{array}$ & $\begin{array}{c}\text { Hyperbolic parabolic } \\
\text { shell }\end{array}$ \\
\hline $\begin{array}{l}\text { Light } \\
3\end{array}$ & Very short & Rather difficult & Cylindrical shell \\
\hline$\underset{3}{\text { Light }}$ & $\begin{array}{c}\text { Very short } \\
4\end{array}$ & $\begin{array}{c}\text { Easy } \\
3\end{array}$ & Geodesic dome \\
\hline $\begin{array}{c}\text { Very heavy } \\
1\end{array}$ & $\underset{2}{\text { Long }}$ & $\underset{3}{\text { Easy }}$ & Geodesic dome \\
\hline
\end{tabular}




\begin{tabular}{|c|c|c|c|}
\hline Capacity of re-use & Exterior design & Interior design & Types of structures \\
\hline Possible & Attractive & $\begin{array}{r}\text { Very attractive }_{3} \\
{ }_{3}\end{array}$ & Space frame \\
\hline No possible & Attractive & Attractive & Pneumatic \\
\hline About possible & Very attractive & Attractive & Cable \\
\hline No possible & Very attractive & $\begin{array}{r}\text { Very attractive } \\
3\end{array}$ & Folded plate \\
\hline Possible & Very attractive & $\begin{array}{l}\text { Very attractive } \\
3\end{array}$ & Membrane \\
\hline Possible & $\begin{array}{l}\text { Very attractive } \\
3\end{array}$ & $\begin{array}{l}\text { Very attractive } \\
3\end{array}$ & Tensegrity \\
\hline No possible & Quit attractive & Quit attractive & Arch \\
\hline No possible & Attractive & $\begin{array}{l}\text { Attractive } \\
\end{array}$ & Vault \\
\hline Possible & Quit attractive & Quit attractive & Truss \\
\hline No possible & Quit attractive & Quit attractive & Frame \\
\hline No possible & Very attractive & $\begin{array}{l}\text { Very attractive } \\
3\end{array}$ & $\begin{array}{c}\text { Hyperbolic parabolic } \\
\text { shell }\end{array}$ \\
\hline No possible & Attractive & Attractive & Cylindrical shell \\
\hline Possible & Attractive & $\begin{array}{l}\text { Very attractive } \\
\qquad 3\end{array}$ & Geodesic dome \\
\hline No possible & Quit attractive & Quit attractive & Grid \\
\hline
\end{tabular}

\begin{tabular}{|c|c|c|c|}
\hline Long design life & Flexibility & Demolition & Types of structures \\
\hline High & Flexible & Very easy & Space frame \\
\hline High & Flexible & Easy & Pneumatic \\
\hline Very high & Very flexible & Normally & Cable \\
\hline Very high & Stiff & Difficult & Folded plate \\
\hline High & Very flexible & Easy & Membrane \\
\hline High & Flexible & Very easy & Tensegrity. \\
\hline High & Stiff & Difficult & Arch \\
\hline High & Stiff & Difficult & Vault \\
\hline rather high & Flexible & Easy & Truss \\
\hline High & Stiff & Normally & Frame \\
\hline Very high & Stiff & Difficult & $\begin{array}{c}\text { Hyperbolic parabolic } \\
\text { shell }\end{array}$ \\
\hline Very high & Stiff & Difficult & Cylindrical shell \\
\hline High & Flexible & Very easy & Geodesic dome \\
\hline Very high & Stiff & Difficult & Grid \\
\hline
\end{tabular}




\begin{tabular}{|c|c|c|}
\hline Span & Thickness of roof & $\begin{array}{l}\text { Types of } \\
\text { structures }\end{array}$ \\
\hline $\begin{array}{l}\text { Moderate }=40-60 \mathrm{~m} \\
\text { In stadiums }=160-200 \\
4\end{array}$ & Thickness/span=1/10-1/20 & Space frame \\
\hline $\begin{array}{l}\text { Almost } 200 \mathrm{~m} \\
3\end{array}$ & Air supported: dependence to thickness of materials & Pneumatic \\
\hline $\begin{array}{l}\text { Almost } 100 \mathrm{~m} \\
2\end{array}$ & $\begin{array}{l}\text { Air inflated }=1 / 10-1 / 15 \text { span } \\
\& \text { dependence to length of member and it's curve }\end{array}$ & \\
\hline $\begin{array}{l}200-300 \mathrm{~m} \\
\text { In bridge almost }=2 \mathrm{~km} \\
4\end{array}$ & Depends on finishing covering materials for roof & Cable \\
\hline Almost $100 \mathrm{~m}$ & $\operatorname{Min}=5 \mathrm{~cm}$ & Folded plate \\
\hline 4 & $\begin{array}{l}\text { Most in support bearing points } \\
\text { Height/span=1/5-1/15 }\end{array}$ & \\
\hline $\begin{array}{l}\text { Almost } 40 \mathrm{~m} \\
4\end{array}$ & $\begin{array}{l}\text { Height } / \text { span }=1 / 3-1 / 6 \\
\text { Depends on the curve of membrane }\end{array}$ & Membrane \\
\hline $\begin{array}{l}\text { Almost } 150 \mathrm{~m} \\
3\end{array}$ & Depends on span & Tensegrity \\
\hline Almost 30 m & $\mathrm{H}=\left.\mathrm{w}\right|^{2} / 8 f$ & Arch \\
\hline 1 & $\begin{array}{l}F=\text { rise of arch } \\
L=\text { span } \\
W=\text { versatile load } \\
H=\text { Thickness of roof }\end{array}$ & \\
\hline $\begin{array}{l}\text { In dome=almost } 41 \mathrm{~m} \\
2\end{array}$ & Depends on materials \& span & Vault \\
\hline $\begin{array}{l}\text { In bridges=almost } 1 \mathrm{~km} \\
\text { In other function }=130 \mathrm{~m} \\
3\end{array}$ & $\begin{array}{l}\text { In steel truss }=\text { thickness } / \text { span }=1 / 10-1 / 20 \\
\text { In concrete truss }=\text { thickness } / \text { span }=1 / 10-1 / 12\end{array}$ & Truss \\
\hline $\begin{array}{l}\text { Normal frame }=7-8 \mathrm{~m} \\
1\end{array}$ & Min $=20 \mathrm{~cm}$ & Frame \\
\hline $\begin{array}{l}60 \mathrm{~m} \\
4\end{array}$ & $\begin{array}{l}\text { Min }=5 \mathrm{~cm} \\
\& \text { more in support bearing points... Span/height=1/3-1/6 }\end{array}$ & $\begin{array}{l}\text { Hyperbolic } \\
\text { parabolic shell }\end{array}$ \\
\hline $\begin{array}{l}20-60 m \\
3\end{array}$ & $\operatorname{Min}=5 \mathrm{~cm}$ & Cylindrical shell \\
\hline $\begin{array}{l}\text { In single layer }=60 \mathrm{~m} \\
\text { In multi layer }=300 \mathrm{~m} \\
3\end{array}$ & In single layer $=\min =1 / 10-1 / 20$ diameter & Geodesic dome \\
\hline $60 m$ & & Grid \\
\hline
\end{tabular}




\begin{tabular}{|c|c|c|c|c|}
\hline Dynamic response & & $\begin{array}{l}\text { Reuse(Dismantling } \\
\text { \& erection) }\end{array}$ & Static response & Types of structures \\
\hline No problem & 4 & possible & $\begin{array}{l}\text { Very } \\
\text { satisfactory }\end{array}$ & Space frame \\
\hline Problematic & 1 & No possible & Satisfactory & Pneumatic \\
\hline Problematic & 1 & About possible & Satisfactory & Cable \\
\hline No problem & 4 & No possible & $\begin{array}{l}\text { Very } \\
\text { satisfactory }\end{array}$ & Folded plate \\
\hline Possible problem & 2 & Possible & Satisfactory & Membrane \\
\hline No problem & 4 & Possible & $\begin{array}{l}\text { Very } \\
\text { satisfactory }\end{array}$ & Tensegrity \\
\hline No problem & 4 & No possible & $\begin{array}{l}\text { Almost } \\
\text { satisfactory }\end{array}$ & Arch \\
\hline No problem & 4 & No possible & Satisfactory & Vault \\
\hline Possible problem & 2 & Possible & Satisfactory & Truss \\
\hline Un certain & 3 & No possible & Satisfactory & Frame \\
\hline Un certain & 3 & No possible & Satisfactory & $\begin{array}{c}\text { Hyperbolic parabolic } \\
\text { shell }\end{array}$ \\
\hline No problem & 4 & No possible & Satisfactory & Cylindrical shell \\
\hline No problem & 4 & possible & $\begin{array}{l}\text { Almost } \\
\text { satisfactory }\end{array}$ & Geodesic dome \\
\hline Very high forces caused & 2 & No possible & Satisfactory & Grid \\
\hline
\end{tabular}

\begin{tabular}{|c|c|}
\hline Problems & Types of structures \\
\hline Needs to skillful constituent \& workman & Space frame \\
\hline $\begin{array}{l}\text { Air supported= vibration problem, providing to access, dysfunction of } \\
\text { mechanical facility }\end{array}$ & \multirow[t]{2}{*}{ Pneumatic } \\
\hline Air inflated= rupture for pressure, weariness for more loading & \\
\hline Vibration resultant wind(that is controlled with secondary cables) & Cable \\
\hline molding & Folded plate \\
\hline Vibration resultant wind(that is controlled with secondary cables) & Membrane \\
\hline Control to percentage of tension in cables & Tensegrity \\
\hline $\begin{array}{l}\text { most control to support bearing point, control to bending loads, control to } \\
\text { propulsion }\end{array}$ & Arch \\
\hline $\begin{array}{l}\text { most control to support bearing point, control to bending loads, control to } \\
\text { propulsion }\end{array}$ & Vault \\
\hline Needs to control of thermal exchange, control of moisture & Truss \\
\hline Needs to attenuated performing & Frame \\
\hline Many difficult with formwork casting & $\begin{array}{l}\text { Hyperbolic parabolic } \\
\text { shell }\end{array}$ \\
\hline Molding \& casting & Cylindrical shell \\
\hline Performing problems(moisture, accessibility) & Geodesic dome \\
\hline Heavy structure & Grid \\
\hline
\end{tabular}


Table 3: Scoring structures, taking into account the usage of College of Architecture

\begin{tabular}{|c|c|c|c|c|c|c|c|c|c|c|c|c|c|c|c|}
\hline 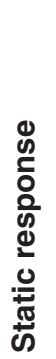 & 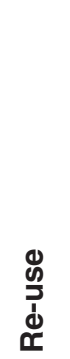 & 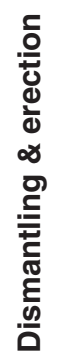 & 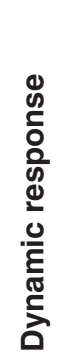 & 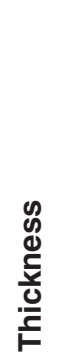 & $\begin{array}{l}\text { ๙ } \\
\text { के }\end{array}$ & 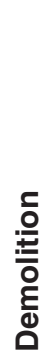 & $\begin{array}{l}\stackrel{\vec{Z}}{\overline{0}} \\
\frac{0}{\mathbb{x}} \\
\frac{0}{4}\end{array}$ & 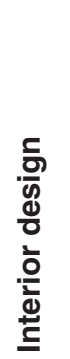 & 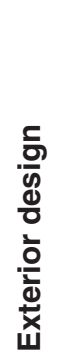 & 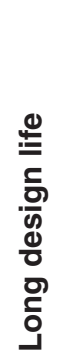 & 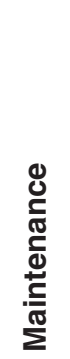 & 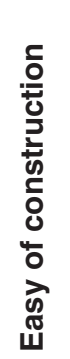 & 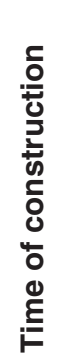 & 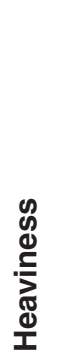 & 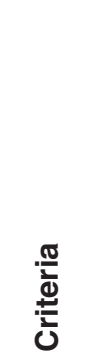 \\
\hline 4 & 1 & 0 & 8 & 0 & 7 & 2 & 5 & 10 & 10 & 7 & 6 & 4 & 4 & 4 & Score \\
\hline
\end{tabular}

Table 4: Scoring structures, taking into account the usage of College of Architecture (First Score is the importance of standards and is of 10 , the second score, is the rating inherent of structures in the relevant field and is of four. The result of multiplying these two numbers is the score of usage in the College of architecture and is also in terms of the desired criterion.)

\begin{tabular}{|c|c|c|c|c|c|c|c|c|c|c|c|c|c|c|}
\hline \multicolumn{2}{|c|}{ Heaviness } & \multicolumn{2}{|c|}{$\begin{array}{l}\text { Time of } \\
\text { constructi } \\
\text { on }\end{array}$} & \multicolumn{2}{|c|}{$\begin{array}{l}\text { Easy of } \\
\text { construction }\end{array}$} & \multicolumn{2}{|c|}{$\begin{array}{l}\text { Capacity } \\
\text { of re-use }\end{array}$} & \multicolumn{2}{|c|}{$\begin{array}{l}\text { Exterior } \\
\text { design }\end{array}$} & \multicolumn{2}{|c|}{$\begin{array}{l}\text { Interior } \\
\text { design }\end{array}$} & \multicolumn{2}{|c|}{$\begin{array}{l}\text { Long design } \\
\text { life }\end{array}$} & \multirow{2}{*}{$\begin{array}{c}\begin{array}{c}\text { Types of } \\
\text { Structures }\end{array} \\
\text { Space frame }\end{array}$} \\
\hline 3 & 4 & 4 & 4 & 4 & 4 & 4 & 1 & 2 & 10 & 3 & 10 & 3 & 7 & \\
\hline \multicolumn{2}{|r|}{12} & & 16 & \multicolumn{2}{|r|}{16} & \multicolumn{2}{|r|}{4} & \multicolumn{2}{|r|}{20} & \multicolumn{2}{|r|}{30} & \multicolumn{2}{|r|}{21} & \multirow{3}{*}{ Pnewnatic } \\
\hline 4 & 4 & 4 & 4 & \multirow{2}{*}{\multicolumn{2}{|c|}{8}} & 3 & 1 & 3 & 10 & 3 & 10 & 3 & 7 & \\
\hline \multicolumn{2}{|r|}{16} & & 16 & & & \multicolumn{2}{|r|}{3} & \multicolumn{2}{|r|}{30} & \multicolumn{2}{|r|}{30} & \multicolumn{2}{|r|}{21} & \\
\hline 4 & 4 & 3 & 4 & 2 & 4 & 3 & 1 & 3 & 10 & 2 & 10 & 4 & 7 & \multirow[t]{2}{*}{ Cable } \\
\hline \multicolumn{2}{|r|}{16} & & 12 & \multicolumn{2}{|r|}{8} & \multicolumn{2}{|r|}{3} & \multicolumn{2}{|r|}{30} & \multicolumn{2}{|r|}{20} & & 28 & \\
\hline 2 & 4 & 2 & 4 & 3 & 4 & 2 & 1 & 3 & 10 & 3 & 10 & 4 & 7 & Folded plate \\
\hline & 8 & & 8 & & 12 & & 2 & & 30 & & 30 & & 28 & \\
\hline 4 & 4 & 3 & 4 & 2 & 4 & 4 & 1 & 3 & 10 & 3 & 10 & 3 & 7 & Membrane \\
\hline & 16 & & 12 & & 8 & & 4 & & 30 & & 30 & & 21 & \\
\hline 3 & 4 & 1 & 4 & 1 & 4 & 4 & 1 & 3 & 10 & 3 & 10 & 3 & 7 & Tensegity \\
\hline & 12 & & 4 & & 4 & & 4 & & 30 & & 30 & & 21 & \\
\hline 1 & 4 & 2 & 4 & 2 & 4 & 2 & 1 & 1 & 10 & 1 & 10 & 3 & 7 & Arch \\
\hline & 4 & & 8 & & 8 & & 2 & & 10 & & 10 & & 21 & \\
\hline 1 & 4 & 2 & 4 & 2 & 4 & 2 & 1 & 2 & 10 & 2 & 10 & 3 & 7 & Vault \\
\hline & 4 & & 8 & & 8 & & 2 & & 20 & & 20 & & 21 & \\
\hline 3 & 4 & 4 & 4 & 4 & 4 & 4 & 1 & 1 & 10 & 1 & 10 & 2 & 7 & Truss \\
\hline & 12 & & 16 & & 16 & & 4 & & 10 & & 10 & & 14 & \\
\hline 2 & 4 & 3 & 4 & 2 & 4 & 2 & 1 & 1 & 10 & 1 & 10 & 3 & 7 & Frame \\
\hline & 8 & & 12 & & 8 & & 2 & & 10 & & 10 & & 21 & \\
\hline 2 & 4 & 2 & 4 & 1 & 4 & 2 & 1 & 3 & 10 & 3 & 10 & 4 & 7 & Hyperbolic \\
\hline & 8 & & 8 & & 4 & & 2 & & 30 & & 30 & & 28 & $\begin{array}{c}\text { parabolic } \\
\text { shell }\end{array}$ \\
\hline 1 & 4 & 2 & 4 & 2 & 4 & 2 & 1 & 2 & 10 & 2 & 10 & 4 & 7 & Cylindrical \\
\hline & 4 & & 8 & & 8 & & 2 & & 20 & & 20 & & 28 & shell \\
\hline 3 & 4 & 4 & 4 & 3 & 4 & 4 & 1 & 3 & 10 & 3 & 10 & 3 & 7 & Geodesic \\
\hline & 12 & & 16 & & 12 & & 4 & & 30 & & 30 & & 21 & dome \\
\hline 1 & 4 & 2 & 4 & 3 & 4 & 2 & 1 & 1 & 10 & 1 & 10 & 4 & 7 & Grid \\
\hline & 4 & & 8 & & 12 & & 2 & & 10 & & 10 & & 28 & \\
\hline
\end{tabular}




\begin{tabular}{|c|c|c|c|c|c|c|c|c|c|c|}
\hline \multirow{2}{*}{$\begin{array}{l}\begin{array}{l}\text { Types of } \\
\text { Demolition }\end{array} \\
\text { Space frame }\end{array}$} & \multicolumn{2}{|c|}{$\begin{array}{l}\text { Flexibility } \\
\text { Structures }\end{array}$} & \multicolumn{3}{|c|}{$\begin{array}{l}\text { Static } \\
\quad \text { response }\end{array}$} & \multicolumn{2}{|c|}{$\begin{array}{l}\text { Dynamic } \\
\text { response }\end{array}$} & \multicolumn{2}{|c|}{ Span } & \multirow[b]{2}{*}{4} \\
\hline & 5 & 3 & 4 & 4 & 8 & 4 & 7 & 4 & 2 & \\
\hline & 15 & & 16 & & 32 & & 28 & & 8 & \\
\hline Pneumatic & 5 & 3 & 4 & 3 & 8 & 1 & 7 & 3 & 2 & 3 \\
\hline \multirow[t]{3}{*}{ Cable } & 15 & & & & & & 21 & & & \\
\hline & 5 & 4 & 12 & & 8 & & 7 & 2 & 6 & \\
\hline & 20 & & & & & & 14 & & & \\
\hline \multirow[t]{2}{*}{ Cable } & 5 & 2 & 4 & 3 & 8 & 1 & 7 & 4 & 2 & 3 \\
\hline & 10 & & 12 & & 8 & & 28 & & 6 & \\
\hline \multirow[t]{2}{*}{ Folded plate } & 5 & 4 & 4 & 4 & 8 & 4 & 7 & 4 & 2 & 1 \\
\hline & 20 & & 16 & & 32 & & 28 & & 2 & \\
\hline \multirow[t]{2}{*}{ Membrane } & 5 & 3 & 4 & 3 & 8 & 2 & 7 & 4 & 2 & 3 \\
\hline & 15 & & 12 & & 16 & & 28 & & 6 & \\
\hline \multirow[t]{2}{*}{ Tensegrity } & 5 & 2 & 4 & 4 & 8 & 4 & 7 & 3 & 2 & 4 \\
\hline & 10 & & 16 & & 32 & & 21 & & 8 & \\
\hline \multirow[t]{2}{*}{ Arch } & 5 & 2 & 4 & 2 & 8 & 4 & 7 & 1 & 2 & 1 \\
\hline & 10 & & 8 & & 32 & & 7 & & 2 & \\
\hline \multirow[t]{2}{*}{ Vault } & 5 & 3 & 4 & 2 & 8 & 4 & 7 & 2 & 2 & 1 \\
\hline & 15 & & 8 & & 32 & & 14 & & 2 & \\
\hline \multirow[t]{2}{*}{ Truss } & 5 & 2 & 4 & 3 & 8 & 2 & 7 & 3 & 2 & 3 \\
\hline & 10 & & 12 & & 16 & & 21 & & 6 & \\
\hline \multirow[t]{2}{*}{ Frame } & 5 & 2 & 4 & 3 & 8 & 3 & 7 & 1 & 2 & 3 \\
\hline & 10 & & 12 & & 24 & & 7 & & 6 & \\
\hline \multirow{4}{*}{$\begin{array}{l}\text { Hyperbolic } \\
\text { parabolic shell } \\
\text { Cylindrical shell }\end{array}$} & 5 & 2 & 4 & 3 & 8 & 3 & 7 & 4 & 2 & 1 \\
\hline & 10 & & 12 & & 24 & & 28 & & 2 & \\
\hline & 5 & 2 & 4 & 3 & 8 & 4 & 7 & 3 & 2 & 1 \\
\hline & 10 & & 12 & & 32 & & 21 & & 2 & \\
\hline \multirow[t]{2}{*}{ Geodesic dome } & 5 & 3 & 4 & 2 & 8 & 4 & 7 & 3 & 2 & 4 \\
\hline & 15 & & 8 & & 32 & & 21 & & 8 & \\
\hline \multirow[t]{2}{*}{ Grid } & 5 & 2 & 4 & 3 & 8 & 2 & 7 & 2 & 2 & 1 \\
\hline & 10 & & 12 & & 16 & & 14 & & 2 & \\
\hline
\end{tabular}

issues would occur. So, after specifying the needed criteria and qualities, scoring and quantifying them will be discussed.

Points and credits of each structure is determined with the score and the number of 4 . The four points are interpreted as:

$$
\begin{aligned}
& 1=\text { acceptable } 2=\text { average } \quad 3=\text { Good } \\
& 4=\text { Very Good }
\end{aligned}
$$

In this section the attempt is made to 14 main new structures known in the form of weighted criteria, time of construction, ease of construction, maintenance, durable, beauty of external form, internal form, flexibility, destruction, crater, roof thickness, dynamic forces, the possibility of dismantling and reinstallation, reusability and static forces are rated. (table 2)

\section{Scoring structures based on their specific useges}

The process which has been done in the last stage is done by just considering one usage. In order to better understand the proposed solution for selection of the optimal system the usage of college of architecture is taken in to consideration.

Scoring the most important criteria in Architecture, for the use of college of architecture

In the table below the most important criteria of designing architectural projects and its 
Table 5: Final score of the structures

\begin{tabular}{lccccccc}
\hline $\begin{array}{l}\text { Types of } \\
\text { structures }\end{array}$ & $\begin{array}{l}\text { Space } \\
\text { frame }\end{array}$ & Pneumatic & Cable & $\begin{array}{c}\text { Folded } \\
\text { plates }\end{array}$ & Membrane & Tensegrity & Arch \\
\hline Total of score & 236 & 191 & 203 & 230 & 221 & 205 & 134 \\
\hline $\begin{array}{l}\text { Types of } \\
\text { structures }\end{array}$ & Vault & Truss & Frame & $\begin{array}{c}\text { Hyperbolic } \\
\text { parabolic } \\
\text { shell }\end{array}$ & $\begin{array}{c}\text { Cylindrical } \\
\text { shell }\end{array}$ & $\begin{array}{c}\text { Geodesic } \\
\text { dome }\end{array}$ & Grid \\
\hline Total of score & 161 & 170 & 148 & 210 & 191 & 227 & 150 \\
\hline
\end{tabular}

impact in the design of the College of Architecture is specified. Entered scores are as numbers between $1-10$ that is seen as follows:

\section{Scoring structures, taking into account the usage of College of Architecture Final scoring}

In this table, the sum of the scores obtained by multiplying columns 1 and 2 can be seen. According to the process in 1-5 items about particular use in the College of Architecture, the space frame structure is of priority. Anda folded plates structures has the relative desirability.

\section{Conclusion}

Today, the use of super-structures that are presented by architects, is perhaps because of strong understanding of the structure and aesthetic architecture that is used by architects (Hashempur, 2012). Also, today in most countries with the developed industrial structure and rich and powerful architecture, architectural and structural effects are complementary and together (Akbari, 2012). However, Iranian architecture in the past two decades is the non-schooled architecture, that has no genre or theory and is not clearly articulated so it is disable to compete with the leading world projects is not even comparable to them. One of the things that has created this condition is certainly weak technology and badly executed building. (AfsharNaderi, 2000)

To achieve real progress in the field of architectural materials and our limitations have to carefully consider free from prejudice. (AfsharNaderi, 2000) Power and progress of every society requires power to improve the features and get optimized supplies and modern sociology has completely cleared this matter that economic, scientific and technological development is with cultural aspirations. The importance of having the power to compete with the culture and the society is not possible unless we make it possible to have access to all the technical tools in order to help our construction. (Diba, 2007)

In the modern society of Iran, unfortunately we are suffering from not being up to date with the modern technical and technological tools which are related to construction. Although dealing with reasons of this problem is out of the aim of this article, it is vivid that familiarizing architecture students with modern and new structures can be helpful for our engineers and experts of construction business.

In this article we have tried in a number of table to find reasonable solution and low-error method to select a new structural system among the large number of structures, and also with providing simple and understandable ways with quantifying method, the quality issues we face in the architecture, fear of lack of control over the concepts of new structure fade away. In addition to that, with this method designer would consider wide range of different choices and rich data structures and will be able to choose the optimal structure for the aimed utilization. 
Although the structure of building and its function may look different, structure always has a decisive influence on the architecture of the building. Technical skills and knowledge of construction's science, and its relationship with architecture is a crucial subject. (Zarkesh, 2006) It is clear that if in this era of the separation of construction and art, the attempt be made to answer desired criteria of architectures with using modern and new structures, we can have a building which will last for a long time that we have seen such structures in developed countries.

\section{REFERENCES}

1. Heydary, A.A., Farrokhzad, M., Air supported structures, Journal of architecture and urban designing, 7: (2012).

2. Asefy, M., lamny, E., The challenges of new technologies in architecture and its interaction with the values of Islamic architecture, Journal of BagheNazar, 21: (2013).

3. Golabchi, M., A knowledge-based expert system for selection of appropriate structural systems for large spans, Asian journal of civil engineering(building and housing), 9:179191:(2008).

4. Golabchi, M., The Art of Structural Engineering, international association for bridge and structural engineering symposium, structures for the future, the search for quality, Brazil, (1999).

5. Hesami, S., AkbariKaffash, E., TaheriAmiri, M.J., Selection of the appropriate structural system of modern and traditional systems, using Analytical Hierarchy Process AHP, The second International Conference on Engineering and Construction Management, University of poly-technique, (2013).
6. Hashempur, R., Red lines and similar points of architecture and structure, The second International Conference on Engineering and Construction Management, (2012).

7. Akbari, A., Alirezaee, M.R., Ebrahimi, M., The use of structural elements in architectural design, The second International Conference on Engineering and Construction Management, University of Tehran, (2012).

8. AfsharNaderi, K., The role of the architect, Congress of Architectural and urban designing History, (2000).

9. AfsharNaderi, K., Modern architecture of Iran and development issue, Congress of Architectural and urban designing History, (2000).

10. Diba, D., Taghi-Zadeh, K., Contemporary architecture in Iran and the need to achieve matching technology, Journal of Architecture and city designing, 84: (2007).

11. Zarkesh, A., quality of education and professional work to create harmony between architectural space and structure in the contemporary architecture of West, Journal of Fine Arts, (2006). 